\title{
Estudio microestructural y de resistencia de uniones soldadas de la aleación AW7020 por procedimiento MIG en función de la preparación de bordes( ${ }^{(*)}$
}

\author{
Carlos Alberto Bloem*, $\mathrm{M}^{\mathrm{a}}$ Dolores Salvador**, Vicente Amigó $\sigma^{* *}$ y Angel Vicente
}

\begin{abstract}
Resumen Las aleaciones de aluminio de la serie AW7XXX de medias prestaciones se están empleando ampliamente en el sector del transporte, así como en la construcción de estructuras soldadas. La posibilidad de que estas aleacionés se empleen con mayor profusión está condicionada a un mayor conocimiento del comportamiento de la unión soldada. La evolución de la microdureza como un indicador de resistencia es ampliamente utilizado, pero no hay acuerdo sobre el efecto de la preparación de bordes previo a la soldadura y es por ello que el objetivo de este trabajo sea estudiar la influencia de la preparación de bordes sobre el perfil de microdureza y microestructura de la unión soldada de una aleación de aluminio AW7020, soldada mediante el proceso MIG, empleando como metal de aporte AW5356.
\end{abstract}

Palabras clave Aleaciones de aluminio. Soldadura. Microdureza. Microestructura.

\section{Microstructural and strength study of MIG welded unions of aluminium alloy AW7020, as a function of joint geometry}

\begin{abstract}
Medium strength AW7XXX aluminium alloys are widely used as welded structures and in transportation. The applications of these alloys are limited by the behavior of the welded joints. There is not an agreement on the joint geometry that must be used on the $5 \mathrm{~mm}$ aluminium welds. The microhardness evolution is one of the most important strength indicator. For this reason, the aim of this work is to study the welded joint geometry influence on microhardness profile and on microstructure of a welded aluminium alloy AW7020 MIG, filled with AW5356.
\end{abstract}

Keywords Aluminium alloys. Welding. Microhardness. Microstructure.

\section{INTRODUCCIÓN}

Cada día se emplea el aluminio y sus aleaciones en mayor grado, lo cual se debe principalmente a su baja relación peso/resistencia, su buen comportamiento frente a la corrosión y al fenómeno de endurecimiento por precipitación, el cual fue descubierto por accidente a principios de siglo por Alfred Wilm ${ }^{[1]}$.

El uso del aluminio ha estado restringido a pequeñas piezas o a piezas que no requerían de la soldadura para su ensamble, debido a que la soldabilidad del aluminio no es del todo satisfactoria; por ello se han desarrollado en el mundo un sinnúmero de trabajos relacionados con la soldadura de las aleaciones de aluminio.
Muchos investigadores han profundizado en el estudio de las características mecánicas de la unión soldada $^{[2]}$, o se han dedicado a la fenomenología metalúrgica ${ }^{[3-5]}$. Hay quienes han realizado comparaciones entre diferentes métodos de soldeo ${ }^{[6,7]} y$ otros se han dedicado a los tratamientos post-sol$\mathrm{deo}^{[8]}$, pero muy pocas investigaciones han estudiado la preparación mecánica antes y después del soldeo. Además, el aporte térmico inherente al proceso de soldeo, afecta sustancialmente la microestructura y sus propiedades en los alrededores de la soldadura, zona afectada por el calor (ZAC). Por ello, el objetivo del presente trabajo es cualificar, mediante la evolución de la microdureza en la ZAC, la bondad en la preparación de bordes antes del soldeo.

(•) Trabajo recibido el día 28 de septiembre de 1999 y aceptado en su forma final el 1 de febrero de 2000.

(*) Facultad de Ingeniería. Escuela de Ingeniería Mecánica. Universidad de Los Andes. Mérida (Venezuela).

(**) Dpto. de Ingeniería Mecánica y Materiales. Universidad Politécnica de Valencia. Camino de Vera s/n. 46022 Valencia (España). Tel.: 963877623. Fax:96387 76 29.E-mail:dsalva@mcm.upv.es 
El proceso del soldeo del aluminio con mayor aceptación en el ámbito industrial es el GMAW (MIG) por su excelente calidad, su bajo coste y la ventaja de la ausencia de fundentes y/o protectores sólidos que a la larga pueden perjudicar la calidad de la unión soldada ${ }^{[7]}$.

De las aleaciones de aluminio tratables térmicamente, las de la serie 7XXX poseen una soldabilidad deficiente en aquellas aleaciones cuyo contenido de aleantes total $\mathrm{Zn}+\mathrm{Mg}+\mathrm{Cu}$ es superior al 9 $\%$, pero si este contenido es inferior al $6 \%$, su soldabilidad es elevada ${ }^{[9]}$.

Debido a su buena soldabilidad, las aleaciones como la AW7005, AW7020 y AW7039 se emplean de manera importante en estructuras soldadas.

Sin embargo, el ASM "Specialty handbook. Aluminum and aluminum alloys" ${ }^{\text {"10] }}$ recomienda el empleo de la preparación de bordes a partir de los $5 \mathrm{~mm}$, mientras otras fuentes ${ }^{[10]}$ recomiendan lo mismo a partir de los $8 \mathrm{~mm}$. No obstante, algunos investigadores ${ }^{[11]}$ realizan preparaciones de borde sobre placas con espesores muy inferiores a los anteriormente mencionados. Por todo ello, resulta necesario estudiar el efecto de la preparación o no de bordes en planchas de $5 \mathrm{~mm}$ de espesor o inferiores, así como el efecto que la preparación de bordes puede tener sobre la ZAC en las aleaciones de aluminio de la serie 7XXX, en las que no se ha estudiado con profundidad.

La selección de la aleación se basa en la aplicabilidad de ésta a la industria del transporte terrestre, que siendo uno de los sectores de mayor productividad, es quizás el más reacio a la aplicación de la ligereza del aluminio en sus diseños, lo que se puede entender por ese antiguo mito de que "el aluminio no se puede soldar"; por ello, considerando las prestaciones necesarias en este sector se decide realizar el estudio sobre una aleación de prestaciones medias como la AW7020, que entre otras características posee una soldabilidad aceptable y un envejecimiento natural acelerado.

\section{PROCEDIMIENTO EXPERIMENTAL}

\subsection{Materiales}

El material base de estudio procede de una plancha de AW7020 T6 suministrada por Alumafel, obtenida por laminación, cuya composición química se muestra en la tabla I.

Diferentes fuentes ${ }^{[10,11]}$ recomiendan como material de aporte la aleación de Al-Mg con denominación AW5356, en forma de alambre de 1,2 mm
Tabla I. Composición química (\% en masa)

Table I. Chemical composition (\% mass)

\begin{tabular}{lcccccc}
\hline & Mg & Si & Cr & Mn & Zn & Fe \\
\hline Mat. base AW7020 & 1,2 & 0,17 & 0,31 & 0,20 & 5,05 & 0,25 \\
\hline Aporte AW5356 & 5,0 & - & 0,12 & 0,15 & 0,1 & - \\
\hline
\end{tabular}

de diámetro, cuya composición química se indica también en la tabla I.

\subsection{Soldeo}

Se realizaron uniones soldadas sobre cuatro placas, cuyas dimensiones eran de $350 \times 150 \times 5 \mathrm{~mm}$, dos de ellas se soldaron con preparación de bordes en bisel a $\pm 30^{\circ}$ mientras que las otras fueron a tope, ambas se soldaron a dos pasadas en sentido perpendicular a la dirección de laminación; entre la primera y segunda pasada se realizó una limpieza mecánica con disco abrasivo. El procedimiento de soldeo fue MIG-DC, empleando como gas protector la mezcla $75 \%$ Ar $25 \%$ He. Las condiciones de soldeo se recogen en la tabla II.

La preparación de bordes, a las probetas soldadas con bisel, se realizó mediante muela abrasiva dándole un ángulo de $\pm 30^{\circ}$, justo antes del soldeo; la limpieza de las juntas a soldar se realizó mediante un suave cepillado con disco de cerdas de acero inoxidable, manteniendo la morfología de la junta; la separación del talón empleado fue de 0,5 $\mathrm{mm}$ en el caso de las biseladas y $1 \mathrm{~mm}$ en el caso de las soldadas a tope, además con la finalidad de mantener la distancia y el paralelismo entre las placas durante el proceso de soldadura, éstas se ajustaron a un par de caballetes.

Los sobrecordones se eliminaron en su totalidad mediante muela abrasiva para minimizar

Tabla II. Condiciones de soldeo

Table II. Welding parameters

\begin{tabular}{lccccc}
\hline Condición & Ángulo & $V(\mathrm{~V})$ & $/(\mathrm{A})$ & $\begin{array}{c}\text { Vel. } \\
(\mathrm{mm} / \mathrm{min})\end{array}$ & $\begin{array}{c}\mathrm{EBA} \\
(\mathrm{kJ} / \mathrm{cm})\end{array}$ \\
\hline Sin bisel & - & 21,0 & 140 & $525^{*}$ & 5,6 \\
& & & & $382^{* *}$ & 7,7 \\
Con bisel & $\pm 30^{\circ}$ & 19,3 & 120 & $412^{*}$ & 5,6 \\
& & & & $350^{* *}$ & 6,6 \\
\hline
\end{tabular}

* Primera pasada.

** Segunda pasada. 
cualquier efecto que pudiese generar una diferencia de tamaño del sobrecordón.

La calidad de la soldadura se evaluó primero por una inspección óptica, en cuanto a falta de penetración o descuelgues. Posteriormente, se examinaron mediante líquidos penetrantes antes y después de eliminar el sobrecordón y, finalmente, se radiografiaron, descartándose aquellas probetas que presentasen defectos lineales o porosidad superior a los 0,5 $\mathrm{mm}$ de longitud ó 1,0 $\mathrm{mm}$ de diámetro.

\subsection{Estudio metalográfico}

Se prepararon probetas para el estudio metalográfico mediante microscopía óptica (MO) y electrónica de barrido (SEM) con el fin de evaluar posibles diferencias microestructurales entre las dos condiciones de soldeo, con o sin bisel. De igual manera se realizaron análisis cuantitativos mediante microsonda de RX. Los equipos empleados fueron un microscopio Nikon modelo Microphot FX y un microscopio electrónico de barrido (SEM) marca Jeol modelo JSM6300, el cual tiene acoplado un análisis de dispersión de energías de rayos $\mathrm{X}$ (EDX).

\subsection{Caracterización mecánica}

Se realizaron diversos barridos de microdurezas a lo largo de la sección transversal de las probetas soldadas en el cordón y a lo largo de la zona afectada por el calor (ZAC) siguiendo la norma ASTM E384-73 ${ }^{[13]}$, empleando para ello un microdurómetro marca Matsuzawa, modelo MHT2, con un indentador del tipo Vickers, aplicándose una carga de $100 \mathrm{~g}$ durante $15 \mathrm{~s}$.

El patrón de espaciamiento seguido en los barridos de microdureza se asemeja al empleado por Malin $^{[14]}$, con la variante que se evaluó cada 0,1 ; 0,3 y $0,6 \mathrm{~mm}$, como se aprecia en la figura 1 , donde el punto cero o de partida es la interfase entre el cordón (baño fundido) y el metal base; el extremo negativo se encuentra en la zona media del

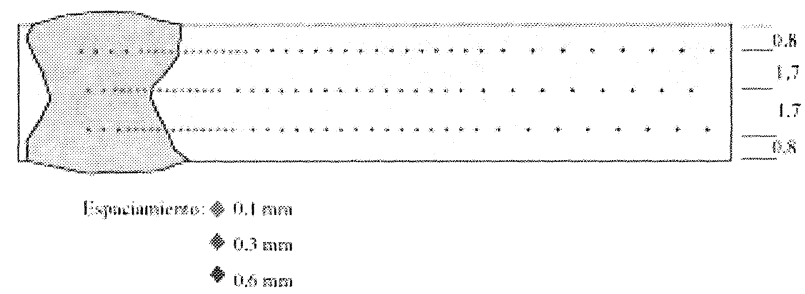

Figura 1. Patrón de espaciamiento en la microdureza.

Figure 1. Microhardness spacing schedule. cordón y el extremo positivo a la distancia de 32 $\mathrm{mm}$.

Con objeto de confirmar los resultados de microdureza, se realizaron ensayos de tracción de acuerdo con la norma UNE 7-474-92, EN 10-0021 , a una velocidad de $5 \mathrm{~mm} / \mathrm{min}$. en una máquina de ensayos universal Instron 4204, recogiéndose en la tabla III el valor promedio de los datos obtenidos.

\section{RESULTADOS Y DISCUSIÓN}

Con el fin de evaluar la variación del tamaño y dimensiones del cordón como una función única del bisel, se midió el tamaño promedio de los cordones mediante la digitalización de imágenes de la sección transversal de las probetas. Las dimensiones se reflejan en la tabla IV y el aspecto macroscópico puede observarse en la figura 2. De las cuales se puede. indicar que el tamaño promedio del cordón realizado sin bisel es ligeramente mayor que el correspondiente con bisel, cuantificado por la mayor área del cordón, teniendo una relación directa con el aporte térmico suministrado como se puede observar en las tablas II y IV. De la misma manera, la dispersión de las dimensiones del

Tabla III. Resultados de los ensayos de tracción

Table III. Axial test results

\begin{tabular}{cccc}
\hline Probeta & $\begin{array}{c}\text { Límite de } \\
\text { elasticidad } \\
0,2 \%(\mathrm{MPa})\end{array}$ & $\begin{array}{c}\text { Tensión de } \\
\text { Rotura } \\
(\mathrm{MPa})\end{array}$ & $\begin{array}{c}\text { Alargamiento } \\
(\%)\end{array}$ \\
\hline Material base & $344,4(100 \%)$ & $392,2(100 \%)$ & $15,37(100 \%)$ \\
$\begin{array}{c}\text { Probetas sin } \\
\text { bisel }\end{array}$ & $195,1(57 \%)$ & $271,6(69 \%)$ & $6,07(40 \%)$ \\
$\begin{array}{c}\text { Probetas con } \\
\text { bisel }\end{array}$ & $229,0(66 \%)$ & $265,9(67 \%)$ & $5,69(37 \%)$ \\
\hline
\end{tabular}

Tabla IV. Dimensiones de los cordones

Table IV. Welded zone dimensions

\begin{tabular}{cccc}
\hline $\begin{array}{c}\text { Preparación } \\
\text { de } \\
\text { bordes }\end{array}$ & $\begin{array}{c}\text { Área } \\
\left(\mathrm{mm}^{2}\right)\end{array}$ & $\begin{array}{c}\text { Área del } \\
\text { sobrecordón }\end{array}$ & $\begin{array}{c}\text { Distancias } \\
\text { máximas } \\
\text { y mínimas }(\mathrm{mm})\end{array}$ \\
\hline Sin bisel & $34,22 \pm 9,8$ & 26,74 & $\begin{array}{c}9,39 \pm 2,1 \\
4,18 \pm 1,8\end{array}$ \\
\hline Bisel $\pm 30^{\circ}$ & $31,21 \pm 5,9$ & 30,91 & $8,93 \pm 1,6$ \\
\hline
\end{tabular}




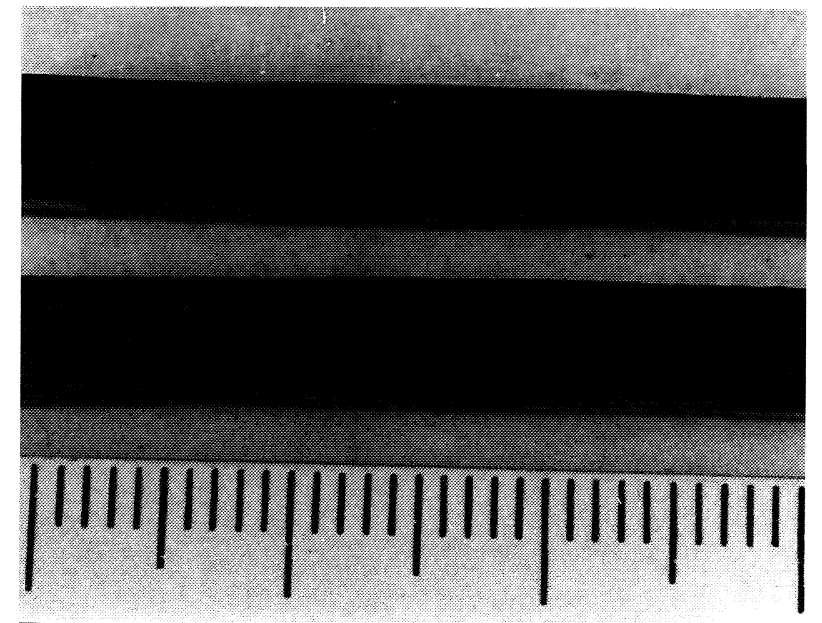

Figura 2. Macrografías de las soldaduras. Probeta superior sin bisel; inferior, con bisel a $\pm 30^{\circ}$. Ataque Weck.

Figure 2. Weld zone macrograph. Upper sample without $\mathrm{V}$-bevel; lower one with $60^{\circ} \mathrm{V}$-bevel. Weck's reagent.

cordón con bisel es considerablemente inferior a las dimensiones de las realizadas sin bisel, de lo que podría inferirse que la repetibilidad de resultados llegue a ser mayor en el caso de las biseladas. No obstante, cabe resaltar que la morfología del cordón en el caso de la soldadura realizada sin biselado de bordes resulta más simétrica en su sentido transversal.

Los resultados de microanálisis por EDX realizados a lo largo de la ZAC demuestran que no se presenta una volatilización de aleantes tal y como indican otros investigadores ${ }^{[7]}$, comprobándose la aparición, en todas las muestras estudiadas, de cantidades relativamente estables de zinc, alrededor del 2,2-3 \% a lo largo del cordón (Fig. 3). Esto hace intuir que durante el proceso de soldeo, la fusión del metal base dentro del cordón es proporcional a la cantidad de material de aporte adicionado, teniendo poca importancia la morfología de la junta; sin embargo se observa que en las

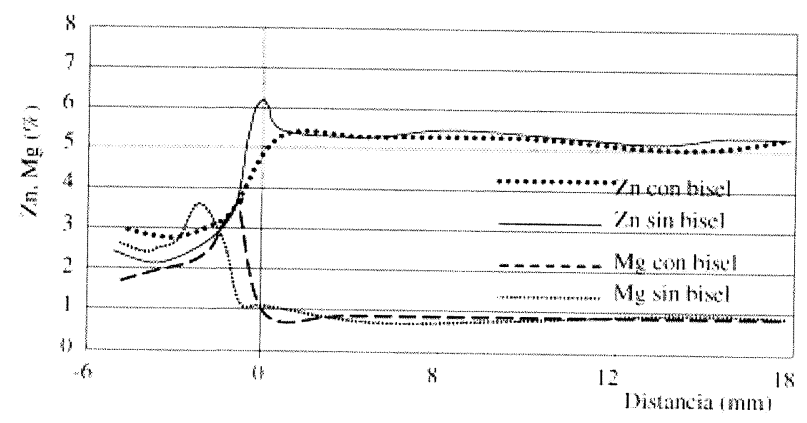

Figura 3. Perfil de la composición de aleantes zinc y magnesio.

Figure 3. Zinc and magnesium composition profile. probetas con bisel la cantidad de zinc es aproximadamente 0,3 puntos superior, lo que implica un incremento del $10 \%$. Los diferentes análisis muestran que se genera dentro del cordón una nueva aleación de la familia Al-Zn-Mg, más pobre en zinc y más rica en magnesio (alrededor del 3 \% Zn y ligeramente inferior al $3 \% \mathrm{Mg}$ ), lo que concuerda con las investigaciones de Yong-Seog ${ }^{[8]}$ y Hussain $^{[15]}$ quien lo corrobora debido a la aparición de precipitados del tipo $\mathrm{T}(\mathrm{Al}, \mathrm{Zn})_{49} \mathrm{Mg}_{32}$.

No obstante, la evaluación microestructural de los dos procesos investigados no muestra cambios apreciables entre ambos, a pesar de que la microscopía óptica con ataque Weck parece mostrar una ZAC ligeramente mayor en las probetas soldadas sin bisel, pero que en todo caso no llega a ser un milímetro mayor que las soldadas con bisel, lo que no es de extrañar debido al ligero incremento de la EBA en las soldadas con bisel.

Como puede observarse en la figura 4, dentro del cordón aparecen pequeñas porosidades, que dada su morfología casi esférica deben ser producto de la liberación de hidrógeno durante la solidificación $^{[10,16]}$.

El estudio microestructural por microscopía óptica muestra cuatro diferentes zonas a lo largo de la interfase. Una primera de granos tipo dendrítico y columnar, posteriormente una zona equiaxial de granos gruesos, seguido de otra zona equiaxial de grano más fino y por último el metal base.

En la zona de granos equiaxiales groseros se observa una mayor definición de los bordes de grano, esta zona es la denominada zonas blancas (ZB) por algunos autores $^{[3]}$. Su tamaño oscila entre los 0,5 y

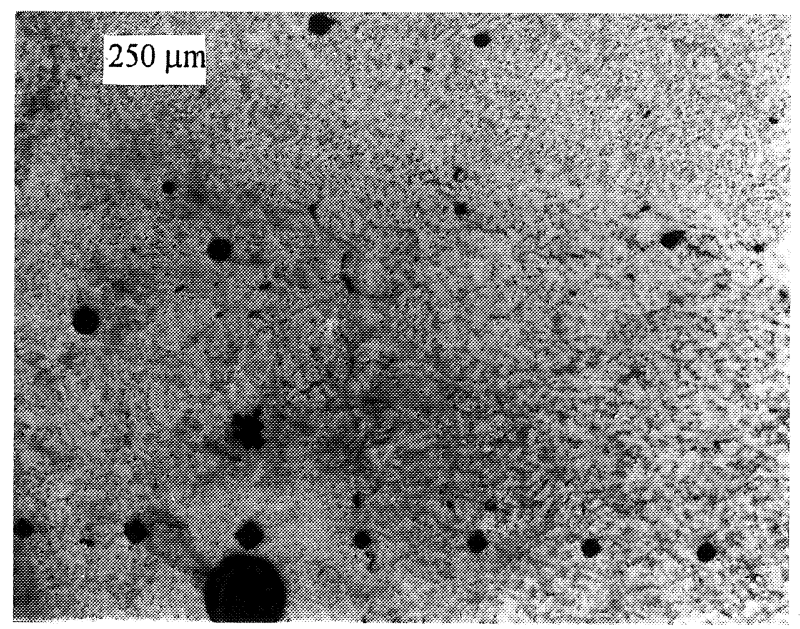

Figura 4. Microestructura de la interfase cordón-metal base. Ataque con Keller x50.

Figure 4. Microstructure of base metal-welded zone interphase. Keller's reagent 50x.

Rev. Metal. Madrid 36 (2000)

http://revistademetalurgia.revistas.csic.es 
$1 \mathrm{~mm}$ y se presenta exclusivamente en la interfase metal base-cordón de la soldadura de las aleaciones de la serie 7XXX, tal como se aprecia en la figura 5 .

Se realizaron tres barridos de microdureza a cada probeta (Fig. 6a) corroborando los valores máximos o mínimos con nuevas mediciones en torno a estos puntos. En la figura $6 \mathrm{~b}$ se muestran los perfiles resultantes en las diferentes zonas de una unión soldada sin bisel. Sin embargo, en la figura 7 se muestran los perfiles representativos de la evolución de la microdureza obtenida a lo largo de la unión soldada con y sin preparación de juntas, desde el baño fundido hasta el metal base.

Como puede observarse, en las figuras $6 \mathrm{~b}$ y 7 se pueden acotar cinco posibles lugares para el fallo mecánico, dos zonas dentro del cordón y tres en la ZAC. En el cordón, el primer lugar se encuentra aproximadamente en el centro con una caída de la dureza en torno al 60-65\% respecto a la del metal base. Esta zona suele desaparecer en el cordón correspondiente a la primera pasada, lo que puede explicarse debido al efecto térmico tan grande que ejerce la segunda pasada sobre el material depositado en la primera, generándose una disolución, homogeneización y hasta precipitación de los elementos dentro del cordón, como lo indica YongSeog ${ }^{[8]}$ basándose en el hecho de que la mezcla del metal base con el material de aporte produce una nueva aleación del tipo Al-Zn-Mg-Mn, como se recoge en los diferentes análisis por microsonda de RX, (Fig. 3).

La segunda zona débil se presenta en una pequeña región que puede oscilar entre 0,2-1 mm dentro del cordón, con una caída gradual en la du-

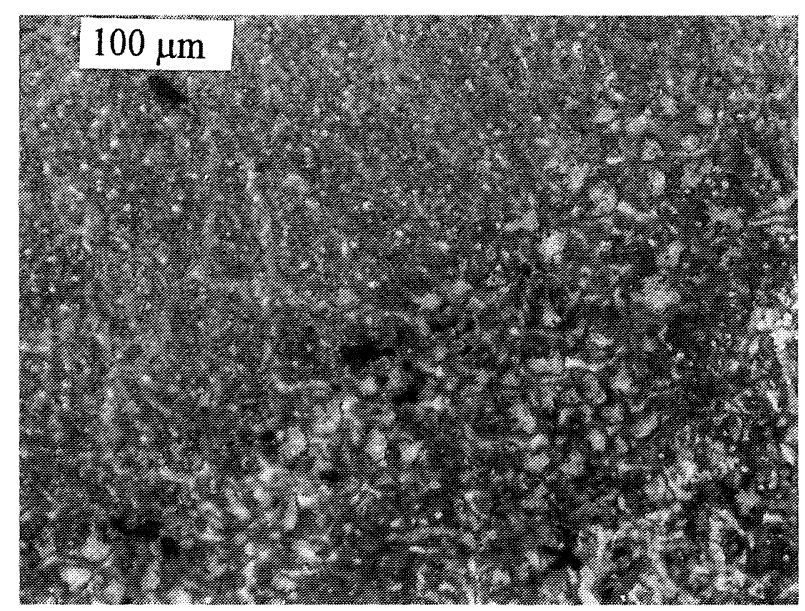

Figura 5. Detalle de la zona blanca en la interfase metal base cordón. Ataque Weck x50.

Figure 5. White zone detail in the welded zone-base metal interphase. Weck's reagent 50x.
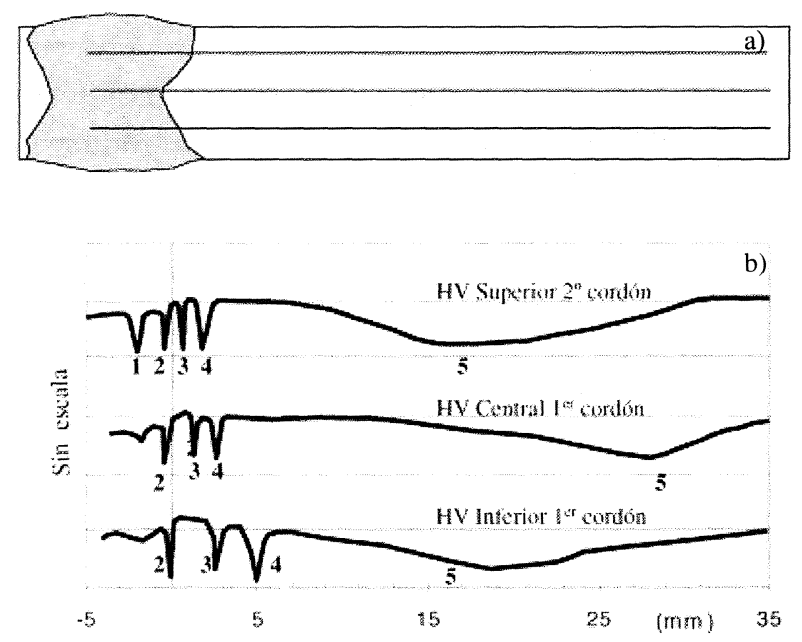

Figura 6. Barrido de microdureza.

a) Esquema del barrido de microdurezas.

b) Evolución de la microdureza en la unión soldada sin preparación de bordes.

Figure 6. Microhardness profile.

a) Schedule of microhardness measurement.

b) Microhardness evolution of the joint without V-bevel preparation.

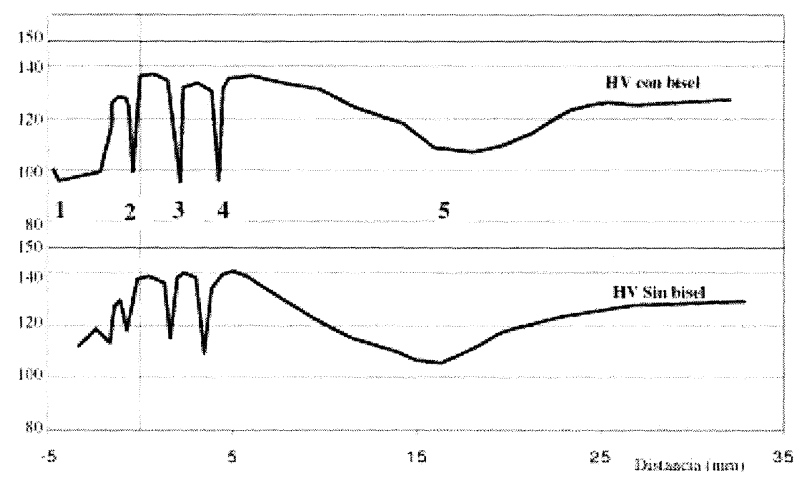

Figura 7. Evolución de las microdurezas del lado superior: $2^{\circ}$ cordón.

Figure 7. Upper side (2nd pass) microhardness evolution.

reza hasta alcanzar un mínimo que en algunos casos puede llegar hasta un $66 \%$ del valor de la dureza del metal base, lo que puede justificarse por las tensiones residuales existentes entre la interfase de los cristales finos y aquellos cristales de carácter dendrítico.

En la ZAC se detecta una tercera zona de descenso de la dureza con valores que en algunos casos pueden llegar a ser del orden del $68 \%$ del valor de la dureza del metal base, la cual se encuentra entre los 0,5 y los $3 \mathrm{~mm}$ desde la línea de fusión. Esta zona estará más alejada cuanto mayor haya sido el aporte térmico durante el proceso de soldeo. 
La cuarta zona corresponde a un descenso importante en la microdureza de iguales características a la anterior, con la que puede confundirse $\mathrm{o}$, en algunos casos, solaparse con la anterior. Su posición se encuentra entre 1,5 y $5 \mathrm{~mm}$ desde la línea de fusión. Esta y la anterior pueden ser el efecto de un fenómeno de solubilización total o parcial durante el proceso de soldeo con una escasa difusión de los elementos aleantes, donde el posterior efecto térmico por la capacitancia térmica genera una precipitación no homogénea de las segundas fases.

Se comprueba la existencia de una quinta zona caracterizada por una ligera caída de la microdureza, en torno al $75 \%$ de la dureza del metal base. Esta se presenta en lo que muchos autores consideran la zona no afectada térmicamente. El mínimo valor se observa a unos 15-20 mm de la línea de fusión y es aquí donde suelen ocurrir las fracturas a tracción cuando éstas se producen en lugares alejados del cordón. El incremento de dureza hacia ambos lados de esta zona se perfila muy gradual y poco acentuado, llegando a alcanzar los valores del metal base en el entorno de los $25-30 \mathrm{~mm}$ para nuestras muestras.

Se observan diferencias en cuanto a la evolución de la microdureza a lo ancho de la ZAC en función de la zona analizada (zona superior, central e inferior). Esto podría deberse a que las planchas durante el soldeo estaban en posición horizontal. El proceso de convección en la plancha por el lado inferior está limitado, pues el aire caliente con tendencia a subir queda atrapado formando una capa sin movimiento y sin posibilidad de transferir calor, mientras que por el lado superior, la convección está asegurada.

Observando detenidamente la figura 7 , se puede notar una pequeña tendencia de las probetas realizadas con bisel a presentar las caídas de microdure$z a$, ligeramente a mayor distancia, pero que en todo caso ésta no llega a ser superior a los $0,3 \mathrm{~mm}$ en la tercera zona, a los $0,5 \mathrm{~mm}$ en la cuarta y a los $2 \mathrm{~mm}$ en la quinta, lo que parece contradictorio, pues el aporte de calor durante el soldeo ha sido ligeramente menor en el caso de las biseladas. Por otro lado, las observaciones microscópicas muestran que las preparadas sin bisel presentan una ZAC ligeramente mayor, lo cual podría ser una respuesta del aporte de calor introducido durante el proceso de soldeo.

Los diferentes análisis de EDX no muestran una volatilización o difusión de los elementos aleantes, por lo que se intuye que las caídas de microdureza se deban exclusivamente a procesos de solución y precipitación en los cuatro primeros puntos y a un proceso de sobreenvejecimiento en la quinta zona.
Sin embargo, Malin ${ }^{[14]}$, Lean et al. ${ }^{[12]}$ y otros autores referencian tres zonas preferentes donde fallan las probetas soldadas de aluminio. Estas zonas se encuentran: en el centro del cordón, en la interfase metal base-soldadura y en una zona dentro de la ZAC, pero algo retirada del cordón. Esto se corrobora con los resultados obtenidos, pues podemos considerar que debido a la cercanía existente entre las zonas 2,3 y 4 , que en la mayoría de los casos estudiados no sobrepasan los 2,0-2,5 mm, cabe la posibilidad de confusión a la hora de evaluar la posición del fallo.

Los ensayos de tracción son indicadores directos del comportamiento estático de un material. Los diferentes ensayos realizados muestran una sensible disminución de la resistencia de la unión soldada respecto de la del material base.

Un aspecto digno de mencionar es, en el caso de las probetas sin bisel, la aparente baja respuesta del límite de elasticidad con respecto a las biseladas, pero si se toma en consideración la tensión de rotura y el porcentaje de alargamiento, se observa que, tanto la tensión de rotura como la plasticidad son ligeramente superiores en las probetas sin bisel, presentando así un comportamiento más seguro.

Comparando el límite de elasticidad de las probetas soldadas con los valores obtenidos del metal base, se observa una ligera diferencia entre ambos procedimientos: $57 \%$ para las no biseladas y $66 \%$ para las biseladas, pero si se considera la tensión de rotura éstas son del orden del $69 \%$ para las no biseladas y $67 \%$ para las biseladas, siendo en este caso favorable el comportamiento de las no biseladas. Por otro lado, se observa que el alargamiento disminuye sensiblemente en ambos casos, del orden del 40 y $37 \%$, lo que claramente indica que la deformación se concentra en las regiones más débiles; por último, cabe resaltar que la mayoría de las fracturas se sucedieron entre las zonas 2 y 3 , y las restantes en las cercanías a la zona 1 , que si bien es la zona más débil (un $6 \%$ inferior a las zonas 2 y 3), ésta sólo se presenta en la parte del cordón correspondiente a la segunda pasada. Sin embargo, la relación del descenso de la tensión de rotura respecto de la del metal base guarda una relación directa con el orden de magnitud del descenso de la microdureza.

\section{CONCLUSIONES}

A lo largo de la investigación desarrollada se ha comprobado la gran utilidad de la microdureza como herramienta para evaluar el comportamiento 
de la unión soldada. De los resultados obtenidos se puede concluir que:

- La evolución de la microdureza es similar en los casos estudiados, con y sin bisel en la preparación, a pesar de que en el caso de las probetas soldadas con bisel las caídas de microdureza sean más acentuadas, alrededor del $3 \%$ mayores.

- Se aprecian, no obstante, en los dos casos estudiados, cinco lugares propicios para que suceda el fallo. Aunque puede resultar difícil diferenciar alguno de ellos.

- No se aprecia volatilización de elementos químicos en la ZAC que puedan justificar la disminución de la microdureza en estas zonas. Esta disminución queda justificada por mecanismos de precipitación.

- La morfología del cordón de las probetas soldadas sin bisel es más simétrica que las soldadas con bisel, aunque el área del cordón resulta mayor y las distancias o anchuras del mismo también.

- A diferencia de lo que algunos autores señalan, la zona de afección térmica se extiende más allá de los 25-30 mm, tal como se observa en los barridos de microdureza, encontrándose una zona de debilidad entre los 15 y $20 \mathrm{~mm}$ desde la interfase del cordón

- La preparación de bordes para las chapas estudiadas, de $5 \mathrm{~mm}$ de espesor, no muestra mejora en las propiedades mecánicas finales de la unión soldada, más bien se obtiene una ligera disminución de las mismas de un 2-3\% con respecto a las uniones sin preparación. A esto, podemos añadir la mayor facilidad y economía de la ejecución de uniones sin preparación de bordes.

\section{Agradecimientos}

Agradecemos la colaboración de la empresa GEC ALSTHOM Transportes, S.A. en las operaciones de soldeo de las probetas empleadas en la investigación.

\section{REFERENCIAS}

[1] I.J. Polmear, Overview Mater. Trans. JIM 37 (1996) 12-31.

[2] O. DebBouz y F. Navaï, J. Mater. Sci. 32 (1997) 475482.

[3] R. HermanN, S.S. BiRley y P. Holdway, Mater. Sci. Eng. $A$ (1996) 247-255.

[4] R.Y. HWANG y C.P. CHOU, Scr. Mater. 38 (1998) 215221.

[5] W. HePples, M.C. ThORNTON y N.J.H. HOLROYd, J. Mater. Sci. 27 (1992) 5720-5726.

[6] L.A. Guitterez, G. Neye y E. ZscheCH, Weld. Res. Suppl. Abril (1996) 115-121.

[7] J.M. Gómez de Salazar, A. Ureña, E. Villauriz, S. Manzanedo e I. Barrena. Rev. Metal. Madrid 34 (1998) 276, 280.

[8] Yong-SeOg KIM y Su IL Pyun. Aluminum 59 (1983) 123-127.

[9] L.F. MONDOLFO, Aluminium Alloys: Structure and Properties. Butterworths, London-Boston, 1976.

[10] American Society for Metals. Specialty handbook, Aluminum and aluminum alloys, ASM International, 1993.

[11] Alubook. http://www.alu-info.dk/Html/alulib/modul/ albook40.htm, dic 1999.

[12] P.P. Lean, J.M. Gómez de Salazar y A. Ureña, Soldadura MIG en arco pulsado del material compuesto AA6092/SiC/25p empleando aportes no reforzados (Al-5Mg). Materiales compuestos 99, pp. 109-116.

[13] American Standard for Testing Materials. ASTM Standards, 1991.

[14] V. MALIN, Weld. Res. Suppl. (1995) 305-318.

[15] H.M. Hussain, P.K. GHOSH, P.C. GUPTA y N.B. POTLURI, Weld. Res. Suppl. Julio (1996) 209-215.

[16] I.R. WHITAKER, Tesis Doctoral. University of Nottingham, 1994. 